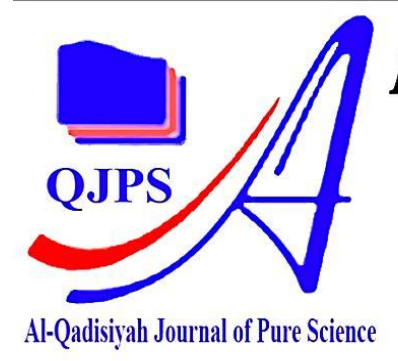

Al-Qadisiyah Journal of Pure Science

ISSN(Printed): 1997-2490ＩSSN(Online): 2411-3514

DOI : /10.29350/jops.

http:// qu.edu.iq/journalsc/ind ex.php/JOPS

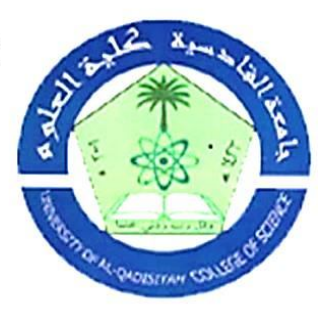

\title{
Numerical Methods for Solving Optimal Control Problem Using Scaling Boubaker Function
}

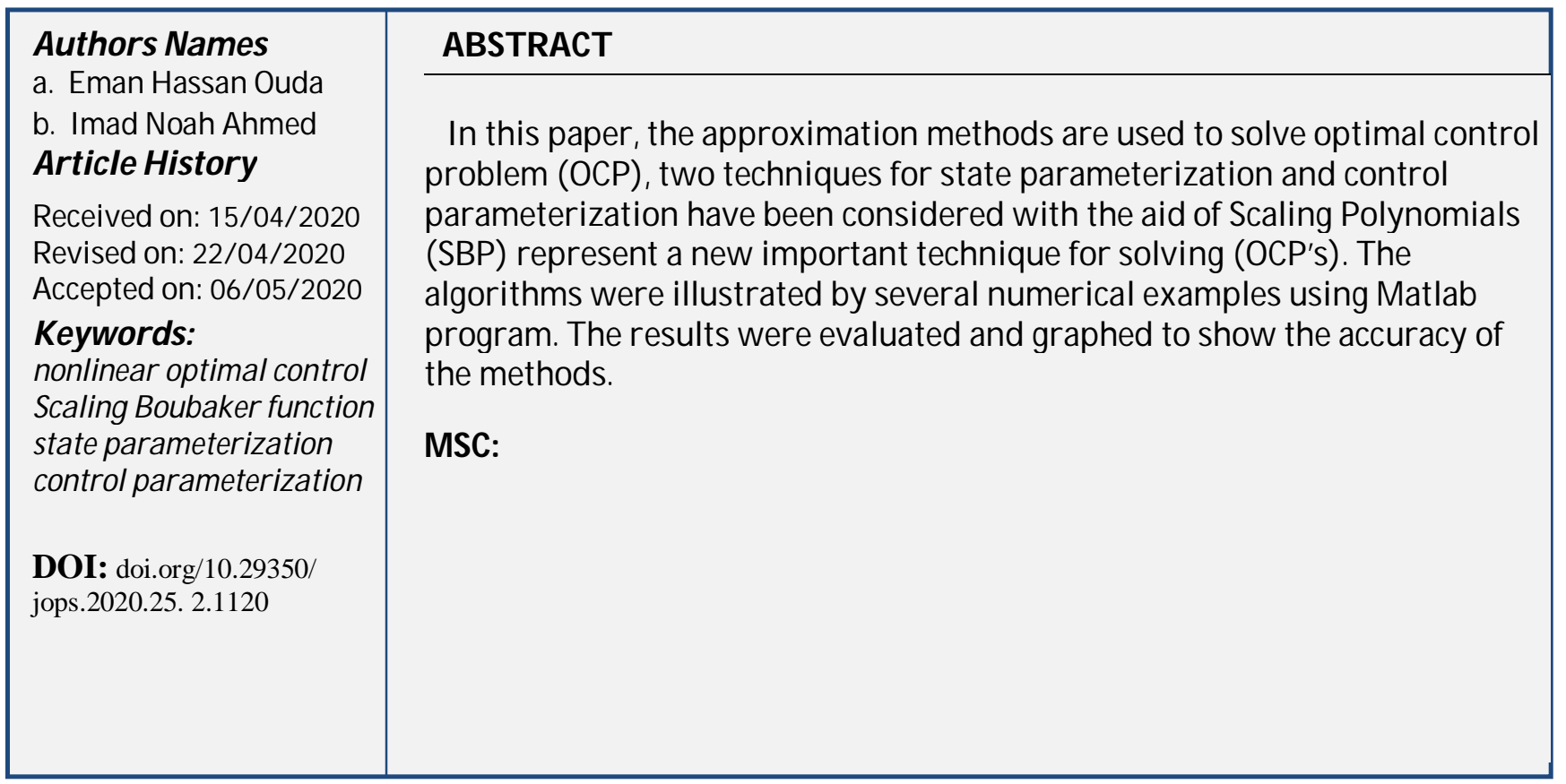

\section{Introduction}

The main idea of the solution process for an optimal control problems is finding a control $u^{*}(t)$ which minimizes a given performance index while satisfying the required constraints. In general, this is done by approximating state or control functions. The finite terms of scaling functions whose unknown coefficients values are sought giving an approximate optimal solution.

Optimal control is a wide field where a lot of researchers used different proceedings for solving its problems. Kafash B. et al. presented new efficient algorithms for solving OCPs and the controlled Duffing oscillator [7]. Lotfi A. Yousefi S. A. and Dehghan M. suggested a numerical direct method for solving a general class of fractional optimal control problems (FOCPs)[10]. Jaddu H.and Majdalawi A. proposed a computational algorithm for solving a class of nonlinear optimal control problems (NOCPs) [6]. Naseif J. and Imad N., used functional analysis technique for studying and deducing sufficient conditions for the controllability of nonlinear boundary value control systems in Banach spaces [12]. Ramezani M. gave a new numerical method for solving (OPC) based on state parameterization and new second kind Chebyshev wavelet [13]. 
Kafash B. and Delavarkhalafi A. introduced an efficient algorithm based on state parameterization solving (OPCs) and Van Der Pol oscillator [8].

The general nonlinear optimal control (NOC) is stated as follows:

$$
\min J=\int_{t_{0}}^{t_{f}} F(t, x(t), u(t)) d t
$$

Subject to the system constraints:

$$
\dot{x}(t)=f(t, x(t), u(t)), \quad t_{0} \leq t \leq t_{f}
$$

with conditions $x\left(t_{0}\right)=x_{0}$

Where $x(t)$ ( the state vector) is $n \times 1$, and $\mathrm{u}(\mathrm{t})$ (the input vector) is $m \times 1$.

The linear quadratic optimal control problem (LQOCP) is a particular form of the general nonlinear (NOC) is as follows:

$$
J=\int_{t_{0}}^{t_{f}}\left(x^{T} Q x(t)+u^{T} R u(t)\right) d t
$$

Subject to the linear

$$
\dot{x}(t)=A x+B u \text { and } x\left(t_{0}\right)=x_{0}
$$

where $\mathrm{Q}(\mathrm{n} \times \mathrm{n})$ and $\mathrm{R}(\mathrm{m} \times \mathrm{m})$ are positive definite matrices. $\mathrm{A}(\mathrm{n} \times \mathrm{n})$, and $\mathrm{B}(\mathrm{m} \times \mathrm{m})$ are constant matrices.

Boubaker polynomials were firstly used as a tool for solving heat equation, then these polynomials were utilized for solving optimal control problems (OCPs) with different proceedings. Kafash B. et al. used Boubaker polynomials with expansion scheme for solving OCPs [9]. Ahmed I., et al utilizing indirect method with Boubaker polynomial for solving an optimal control problem [1].

Goy T. evaluated several families of Toeplitz - Hessenberg determinants whose entries are Boubaker polynomials [4].

Scaling Boubaker polynomials (SBP) and their properties provide powerful mathematical technique for solving some problems in pure and applied mathematics. Scaling functions are widely used in the last decades for dealing with different applied fields in science and engineering. They represent a dilation equation which is a functional of the form

$$
f(t)=\sum_{k=0}^{N} C_{k} f(2 t-K)
$$

with a non - zero solution. Colella D. and Heil Ch. gave a characterization of all dilation equations with continuous and compactly supported solutions [2]. Gradimir V. and Joksimovic D. added some new properties of Boubaker polynomials and applications for achieving an approximate analytical solution of Love's integral equation [5]. Yousfi S. A. presented a numerical solution for the generalized Emden - Fowler equations using Legendre Scaling 
function [14] .Elaydi H., Jaddu H. and Wadi M. used a wavelet approach where the basis orthogonal function were Legendre scaling functions for solving nonlinear optimal control problems[3]. Malmir I. presented an arbitrary scaling parameter for operational matrices of Legendre wavelets [11].

In this paper, two techniques state parameterization and control parameterization have been considered with the aid of scaling Boubaker polynomials (SBP) which represent a new important technique for solving OCP's, the results were evaluated and graphed .

This paper is arranged as follows, in section2, the Boubaker polynomials are introduced, in section3 the Scaling Boubaker polynomials are presented, then in section 4 and 5 the state Parameterization technique and control parameterization technique are explained, in section 6 the convergence of two previous proceedings is studied, then some numerical examples were applied for the proposed method, in section7 comparing the results with exact solutions. Finally the conclusion.

\section{Boubaker polynomials}

Boubaker polynomials Bo have been appeared by Boubaker et al, for solving different equations in many applications ...etc. see [5].

$$
B_{m}(t)=\sum_{s=0}^{\left\lfloor\frac{m}{2}\right\rfloor} \frac{(m-4 s)}{(m-s)}\left(\begin{array}{c}
m-s \\
s
\end{array}\right)(-1)^{s} t^{m-2 s}, \quad \mathrm{~m}=0,1,2, \ldots
$$

The first terms of Boubaker polynomials are

$B_{0}(t)=1, \quad B_{1}(t)=t, \quad B_{2}(t)=t^{2}+2, \ldots$

and the recurrence relation $B_{m}(t)=t B_{m-1}(t)-B_{m-2}(t) \quad, m>2$

\section{Scaling Boubaker polynomials (SBP)}

The Scaling Boubaker polynomials (SBP), can be defined as follows:

$$
S B_{n m}(t)= \begin{cases}2^{\frac{k}{2}} B_{m}\left(2^{k+1} t-2 n-1\right) & \frac{2 n-1}{2^{k+1}} \leq t \leq \frac{2 n}{2^{k+1}} \\ 0 & \text { otherwise }\end{cases}
$$

The arguments of scaling $(k, n, m, t), k$ is positive integer, $n=0,1,2,3, \ldots, 2 k, m$ is degree of Boubaker polynomials and $t$ is the time.

Choosing $\mathrm{k}=1$ and $\mathrm{m}=5$. The first five terms Scaling Boubaker $\mathrm{SB}_{\mathrm{m}}(\mathrm{t})$ were found by using Eq.7 to be:

$$
\begin{aligned}
& S B_{0}=\sqrt{2}, \\
& S B_{1}=\sqrt{2}(4 t-1), \\
& S B_{2}=\sqrt{2}\left(16 t^{2}-8 t+3\right),
\end{aligned}
$$


$S B_{3}=\sqrt{2}\left(64 t^{3}-48 t^{2}+16 t-2\right)$,

$S B_{4}=\sqrt{2}\left(256 t^{4}-256 t^{3}+96 t^{2}-16 t-1\right)$.

The direct methods can be applied by using the parameterization techniques for state variables or control variables or both of them.

\section{The State Parameterization Technique}

The state parameterization is based on approximating state variables by using Scaling Boubaker polynomials (SBP) with unknown coefficients as follows,

$$
x(t)=\sum_{i=0}^{m} a_{i} S B_{i}(t)=\mathrm{a}^{\mathrm{T} S B}(\mathrm{t}) \quad t_{0} \leq t \leq t_{f}
$$

where $a_{i}$ are unknown coefficients of state and SB are the Scaling Boubaker polynomials -Expanding $\mathrm{x}(\mathrm{t})$ using SBP into five order $(\mathrm{m}=5)$ in Eq.7 we get,

$\mathrm{x}(\mathrm{t})=\mathrm{a}_{0} \mathrm{SB}_{0}+\mathrm{a}_{1} \mathrm{SB}_{1}+\mathrm{a}_{2} \mathrm{SB}_{2}+\mathrm{a}_{3} \mathrm{SB}_{3}+\mathrm{a}_{4} \mathrm{SB}_{4}$, where $\mathrm{a}_{\mathrm{i}}$ the state coefficients must be found

with initial condition $x\left(t_{0}\right)=\sum_{i=0}^{4} a_{i} S B_{i}\left(t_{0}\right)=x_{0}$

-Finding $\dot{x}(t)=\sum_{i=0}^{4} a_{i} \dot{S B}_{l}(t) \quad t_{0} \leq t \leq t_{f}$

where the differential of Scaling Boubaker polynomials $\left(S \dot{B}_{\imath}\right)$ is given as follows

$\dot{S B_{0}}(t)=0$

$\dot{S} B_{1}(t)=4 \sqrt{2}$

$\dot{S B_{2}}(t)=\sqrt{2}(32 t-8)$

$\dot{S B} B_{3}(t)=\sqrt{2}\left(192 t^{2}-96 t+16\right)$

$\dot{S} B_{4}(t)=\sqrt{2}\left(1024 t^{3}-768 t^{2}+192 t-16\right)$.

Substituting in state equation (4b) defined by

$$
u(t)=S B(t, x(t), \dot{x}(t))
$$

We get $\mathrm{u}(\mathrm{t})$ which is the control variable

-Deducing the optimal control $u(t)$ with the unknown coefficients of $a_{i}$ we can evaluate $J$ using Eq.1 as in follows 
$J \cong \int_{t_{0}}^{t_{f}}\left(a^{T} S B^{T} Q a S B+a^{T} S B^{T} R a S B\right) d t \quad, t_{0} \leq t \leq t_{f}$

The performance index can written as follows

$$
J=\frac{1}{2} a^{T} H a
$$

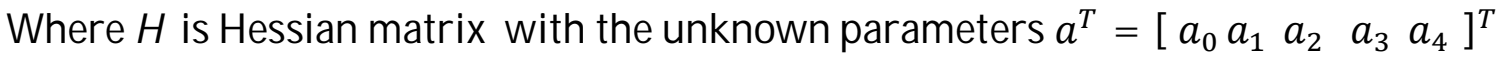
with initial condition using Eq.9 we get the system of equations.

-Solving the system to find coefficients of state.

-Deducing the control, then evaluating the performance function J by Eq.4.

\section{The Control Parameterization Technique}

In this section, the following steps were used to evaluate the control and state by using control parameterization,

- Approximating control variable, as follows

$$
u(t)=\sum_{i=0}^{4} b_{i} S B_{i}, t_{0} \leq t \leq t_{f}
$$

where $b_{i}$ are the unknown coefficients of control and SB are Scaling Boubaker polynomials of order 5 and

- By integrating the state equation system with initial condition we get

$$
\int_{t_{0}}^{t_{f}} u(t) d t=\int_{t_{0}}^{t_{f}} S B(t, x(t), \dot{x}(t)) d t
$$

- Obtaining the solution at the points $\left(t_{n}, t_{n+1}\right)$, the fundamental theorem of calculus can be used, and then Eq.(11) is integrated over [ $\left.t_{n}, t_{n+1}\right]$

- We get the linear algebraic equations which can be solved by Gauss elimination method.

- The coefficients of control $b_{i}$ can be found.

- The state variables can be deduced from the control coefficients by solving state equation.

- Now, substituting $\mathrm{u}(\mathrm{t})$ and $\mathrm{x}(\mathrm{t})$ to evaluate the performance function $\mathrm{J}$ by using Eq.4.

\section{The Convergence Test:}

\subsection{The State Technique:}

$$
x_{m}(t)=\sum_{i=0}^{\infty} a_{i} S B_{i}(t) \quad t_{0} \leq t \leq t_{f}
$$

The truncated series would be

$$
x_{m}(t)=\sum_{i=0}^{m} a_{i} S B_{i}(t)
$$


There using the convergence test for the state parameterization technique.

$\left[\int_{t_{0}}^{t_{f}}\left(x(t)-x_{m}(t)\right)^{2} d t\right]^{\frac{1}{2}}<\varepsilon$

since $\mathrm{x}(\mathrm{t})$ is unknown, it can be replaced the presumably better approximation $\mathrm{x}_{\mathrm{n}+\mathrm{m}}(\mathrm{t})$, where $\mathrm{m} \geq 0$

$\left[\int_{t_{0}}^{t_{f}}\left(x_{n+m}(t)-x_{m}(t)\right)^{2} d t\right]^{\frac{1}{2}}<\varepsilon$

Using the Scaling Boubaker polynomials for approximating the state variables, then

$\left[\int_{t_{0}}^{t_{f}}\left(\sum_{i=0}^{n+m} a_{i} S B_{i}(t)-\sum_{i=0}^{m} a_{i} S B_{i}(t)\right)^{2} d t\right]^{\frac{1}{2}}<\varepsilon$

$=\left[\int_{t_{0}}^{t_{f}}\left(\sum_{i=m+1}^{n+m} a_{i} S B_{i}(t)\right)^{2} d t\right]^{\frac{1}{2}}<\varepsilon$

$=\left[\int_{t_{0}}^{t_{f}}\left(\sum_{i=m+1}^{n+m} a_{i} S B_{i}(t)\right)\left(\sum_{i=m+1}^{n+m} a_{i} S B_{i}(t)\right) d t\right]^{\frac{1}{2}}<\varepsilon$

$=\sum_{i=m+1}^{n+m} \sum_{j=m+1}^{l+m} a_{i} a_{j} \int_{t_{0}}^{t_{f}} S B_{i} S B_{j}(t) d t<\varepsilon$

when the sum of the squares of the remaining coefficients becomes negligible, a satisfactory approximation to the solution is achieved.

\subsection{The Control Technique:}

In the proposed method, the control vector is approximated as,

$u_{m}(t)=\sum_{i=0}^{\infty} b_{i} S B_{i}(t) \quad t_{0} \leq t \leq t_{f}$

The truncated series would be

$u_{m}(t)=\sum_{i=0}^{m} b_{i} S B_{i}(t)$ 
There using the same convergence method as in the previous section (state convergence), we get

$=\sum_{i=m+1}^{n+m} \sum_{j=m+1}^{l+m} b_{i} b_{j} \int_{t_{0}}^{t_{f}} S B_{i} S B_{j}(t) d t<\varepsilon$

when the squares of the remaining coefficients become negligible, a satisfactory approximation to the solution will be reached.

\section{Numerical Examples}

\section{Example 1:}

$\operatorname{Min} j=0.5 \int_{0}^{1}\left(2 x^{2}+u^{2}\right) d t$

with $\dot{x}=0.5 x+u, \quad \mathrm{x}(0)=1$.

where exact solution for this example is $x(t)=\frac{2 e^{3 t}+e^{3}}{e^{\frac{3 t}{2}}\left(2+e^{3}\right)}$, and $u(t)=\frac{2\left(e^{3 t}-e^{3}\right)}{e^{\frac{3 t}{2}}\left(2+e^{3}\right)}$ and the optimal value of $\mathrm{J}=0.8641644977$.

\section{a. Solving by using state parameterization}

By using the state technique illustrated in section 4 , we found the following coefficients $\mathrm{a}_{0}=0.460412396461131, \mathrm{a}_{1}=0.127823658910297, \mathrm{a}_{2}=0.037726555649684$, $\mathrm{a}_{3}=0.003032245966087, \mathrm{a}_{4}=0.000373433066107$.

Then the state and control would be:

$x(t)=1-(919 / 749) t+(937 / 844) t^{2}-(807 / 1970) t^{3}+(353 / 2611) t^{4}$.

and $u(t)=-(1031 / 597)+(2678 / 945) t-(983 / 551) t^{2}+(2336 / 3133) t^{3}-(353 / 5222) t^{4}$.

and Jappro. $=0.86416456896$. With Abs $($ Error $(J))=7.119486900020178 \mathrm{e}-08$.

\section{b. Solving by using control parameterization}

Applying the same example1 by using the control technique illustrated in section 5, we found the following coefficients $\mathrm{b}_{\mathrm{i}} \mathrm{s}$ as follows:

$\mathrm{b}_{0}=-0.680251932678915, \mathrm{~b}_{1}=0.358325053630541, \mathrm{~b}_{2}=-0.055675910199709$, $\mathrm{b}_{3}=0.008291627207229, \mathrm{~b}_{4}=-0.000396395227143$.

we can write approximate solution of state and control as follows:

$\mathrm{x}(\mathrm{t})=(2834 / 61)+(6601 / 307) \mathrm{t}-(2773 / 61) e^{\frac{t}{2}}+(4435 / 652) \mathrm{t}^{2}+(2262 / 4451) \mathrm{t}^{3}+(816 / 2843) \mathrm{t}^{4}$.

$u(t)=-(2089 / 1209)+(779 / 273) t-(4937 / 2631) t^{2}+(312 / 349) t^{3}-(408 / 2843) t^{4}$.

and Jappro. $=0.864160768327565$. With Abs $($ Error $(\mathrm{J}))=3.729433434962459 \mathrm{e}-06$ 
Table1 state results using state Para. And control Para. for example1

\begin{tabular}{|c||c||c|c||c||}
\hline \hline $\mathrm{t}$ & $\begin{array}{c}\mathrm{x}_{\text {appr }}(\mathrm{t}) \text { using } \\
\text { St. Par. }\end{array}$ & $\begin{array}{c}\text { Abs. Error using } \\
\text { St. Par. }\end{array}$ & $\begin{array}{c}\mathrm{x}_{\text {appr }}(\mathrm{t}) \text { using } \\
\text { Co. Par. }\end{array}$ & $\begin{array}{c}\text { Abs. Error using } \\
\text { Co. Par. }\end{array}$ \\
\hline \hline 0 & 0.99999999999 & 0.00000000001 & 1.00000000000 & 0 \\
\hline 0.1 & 0.88800888825 & 0.00003182008 & 0.88798763065 & 0.00001056248 \\
\hline 0.2 & 0.79595296674 & 0.00001814327 & 0.79596996999 & 0.00000114002 \\
\hline 0.3 & 0.72186107669 & 0.00004703036 & 0.72190263669 & 0.00000547036 \\
\hline 0.4 & 0.66408653265 & 0.00003198221 & 0.66411596847 & 0.00000254638 \\
\hline 0.5 & 0.62130712247 & 0.00000749470 & 0.62129891629 & 0.00000071147 \\
\hline 0.6 & 0.59252510732 & 0.00003889426 & 0.59248211274 & 0.00000410030 \\
\hline 0.7 & 0.57706722166 & 0.00003846926 & 0.57702007236 & 0.00000868002 \\
\hline 0.8 & 0.57458467326 & 0.00000587293 & 0.57457247932 & 0.00000632100 \\
\hline 0.9 & 0.58505314321 & 0.00002798628 & 0.58508451573 & 0.00000338623 \\
\hline 1.0 & 0.60877278590 & 0.00000030019 & 0.60876618135 & 0.00000630435 \\
\hline
\end{tabular}

Table2 control results using state Par. And control Par. for example1

\begin{tabular}{|c||c|c||c|c||}
\hline $\mathrm{t}$ & $\begin{array}{c}\mathrm{u}_{\text {appr }}(\mathrm{t}) \mathrm{using} \\
\text { St. Par. }\end{array}$ & $\begin{array}{c}\text { Abs. Error using } \\
\text { St. Par. }\end{array}$ & $\begin{array}{c}\mathrm{u}_{\text {appr }}(\mathrm{t}) \text { using } \\
\text { Co. Par. }\end{array}$ & $\begin{array}{c}\text { Abs. Error using } \\
\text { Co. Par. }\end{array}$ \\
\hline \hline 0 & -1.72696878100 & 0.00136021453 & -1.72787421730 & 0.00045477823 \\
\hline 0.1 & -1.46068395138 & 0.00036649078 & -1.46041120036 & 0.00009373976 \\
\hline 0.2 & -1.22570067102 & 0.00047594913 & -1.22531465614 & 0.00008993425 \\
\hline 0.3 & -1.01778862747 & 0.00003736009 & -1.01773732514 & 0.00001394223 \\
\hline 0.4 & -0.83287974492 & 0.00034044005 & -0.83317637280 & 0.00004381216 \\
\hline 0.5 & -0.66706818424 & 0.00040355034 & -0.66747338954 & 0.00000165494 \\
\hline 0.6 & -0.51661034293 & 0.00015923538 & -0.51681439071 & 0.00004481239 \\
\hline 0.7 & -0.37792485516 & 0.00020830002 & -0.37772981667 & 0.00001326153 \\
\hline 0.8 & -0.24759259173 & 0.00041449041 & -0.24709453271 & 0.00008356860 \\
\hline 0.9 & -0.12235666013 & 0.00014506970 & -0.12212782910 & 0.00008376132 \\
\hline 1.0 & 0.00087759552 & 0.00087759552 & -0.00039342107 & 0.00039342107 \\
\hline \hline
\end{tabular}

Remark: Since the results for the state and control parameterizations are approximately the same, Fig.1 would be sufficient for illustrating the exactness the method. 


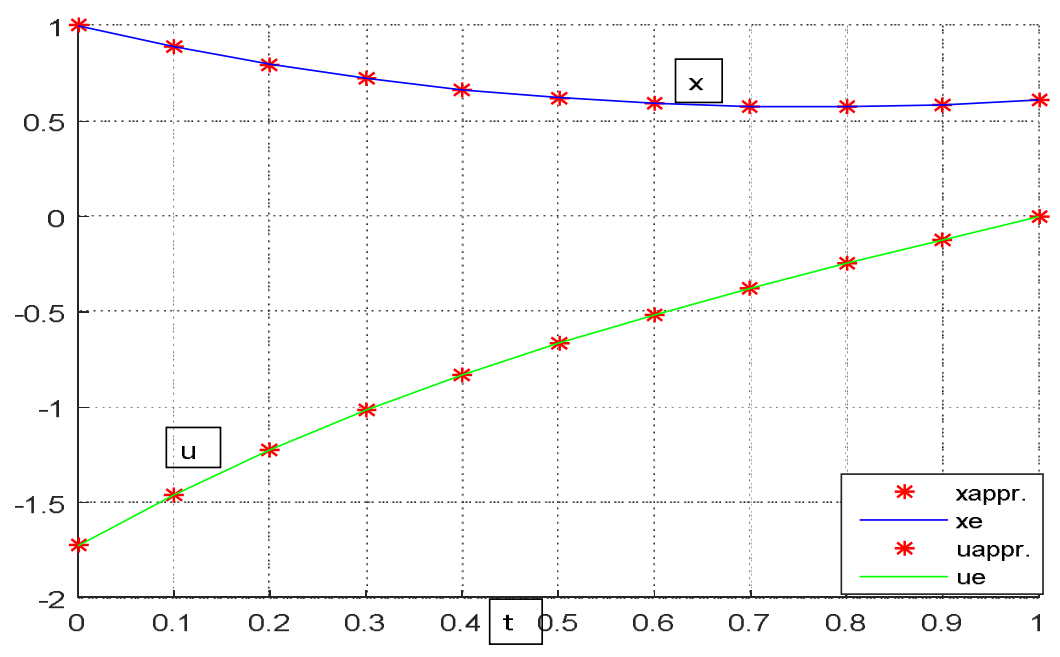

Fig.1 comparison for $\mathrm{x}$ and $\mathrm{u}$ with exact solution for example1

\section{Example 2:}

$\operatorname{Min} j=\int_{0}^{1}\left(\frac{5}{8} x^{2}+\frac{1}{2} x u+\frac{1}{2} u^{2}\right) d t$

with $\dot{x}=0.5 x+u, \quad \mathrm{x}(0)=1$.

where exact solution for this example is $x(t)=\frac{\cosh (1-t)}{\cosh (1)}$, and $u(t)=\frac{-(\tanh (1-t)+0.5)(\cosh (1-t))}{\cosh (1)}$ and the optimal value of Jexact $=0.38797077977882$.

\section{a. Solving by using state parameterization}

The same procedure of state technique in the above example are used to solve this problem we get

$\mathrm{a}_{0}=0.556333395838366, \mathrm{a}_{1}=0.093241205628115, \mathrm{a}_{2}=0.018556405972549$;

$\mathrm{a}_{3}=0.000974119658567, \mathrm{a}_{4}=0.000085277514714$.

Then the state and control would be as follows:

$x(t)=1-(5912 / 7765) t+(1134 / 2279) t^{2}-(427 / 3587) t^{3}+(259 / 8389) t^{4}$.

$u(t)=-(9739 / 7721)+(2211 / 1607) t-(635 / 1048) t^{2}+(528 / 2885) t^{3}-(309 / 20017) t^{4}$.

Jappro $=0.380797080316190$. Abs $($ Error $(\mathrm{J}))=2.338307991678335 \mathrm{e}-09$.

\section{b. Solving by using control parameterization}

Applying the same example2 by using control parameterization, the control with coefficients $b_{i}$ s would be as follows:

$\mathrm{b}_{0}=0.631520147177317, \mathrm{~b}_{1}=0.193446478784298, \mathrm{~b}_{2}=0.021052830981420$,

$\mathrm{b}_{3}=0.001999520525119, \mathrm{~b}_{4}=0.000080517706408$.

$\mathrm{x}(\mathrm{t})=(827 / 90)+(2613 / 784) \mathrm{t}-(737 / 90) e^{\frac{t}{2}}+(629 / 413) \mathrm{t}^{2}+(458 / 9923) \mathrm{t}^{3}+(256 / 4391) \mathrm{t}^{4} ;$ 
$u(t)=(796 / 577) t-(1585 / 2544) t^{2}+(83 / 395) t^{3}-(128 / 4391) t^{4}--(11041 / 8752)$. jappro $=0.380793599989886$. Abs $(\operatorname{Error}(j))=3.477987995992304 \mathrm{e}-06$

Table3 state results using state Para. And control Para. for example2

\begin{tabular}{|c||c|c|c|c|}
\hline $\mathrm{t}$ & $\begin{array}{c}\mathrm{X}_{\text {appr }}(\mathrm{t}) \text { using } \\
\text { St. Par. }\end{array}$ & $\begin{array}{c}\text { Abs. Error using } \\
\text { St. Par. }\end{array}$ & $\begin{array}{c}\text { Xappr }(\mathrm{t}) \text { using } \\
\text { Co. Par. }\end{array}$ & $\begin{array}{c}\text { Abs. Error using } \\
\text { Co. Par. }\end{array}$ \\
\hline 0 & 1.00000000000 & 0.00000000000 & 0.99999344085 & 0.00000655914 \\
\hline 0.1 & 0.92872340268 & 0.00000564606 & 0.92871261917 & 0.00000513744 \\
\hline 0.2 & 0.86672751521 & 0.00000291748 & 0.86672404295 & 0.00000638974 \\
\hline 0.3 & 0.81340923708 & 0.00000840118 & 0.81341084851 & 0.00000678975 \\
\hline 0.4 & 0.76823956480 & 0.00000623615 & 0.76823950787 & 0.00000629308 \\
\hline 0.5 & 0.73076359192 & 0.00000076608 & 0.73075692744 & 0.00000589840 \\
\hline 0.6 & 0.70060050899 & 0.00000693828 & 0.70058739805 & 0.00000617265 \\
\hline 0.7 & 0.67744360360 & 0.00000751209 & 0.67742938850 & 0.00000670300 \\
\hline 0.8 & 0.66106026033 & 0.00000163993 & 0.66105217482 & 0.00000644557 \\
\hline 0.9 & 0.65129196080 & 0.00000528534 & 0.65129229659 & 0.00000494956 \\
\hline 1.0 & 0.64805428366 & 0.00000001000 & 0.64804983167 & 0.00000444198 \\
\hline \hline
\end{tabular}

Table4 control results using state Par. And control Par. for example2

\begin{tabular}{|c|c|c|c|c|}
\hline $\mathrm{t}$ & $\begin{array}{c}\mathrm{u}_{\text {appr }}(\mathrm{t}) \mathrm{using} \\
\text { St. Par. }\end{array}$ & $\begin{array}{c}\text { Abs. Error } \\
\text { using St. Par. }\end{array}$ & $\begin{array}{c}\mathrm{u}_{\text {appr }}(\mathrm{t}) \mathrm{using} \\
\text { Co. Par. }\end{array}$ & $\begin{array}{c}\text { Abs. Error } \\
\text { using Co. Par. }\end{array}$ \\
\hline 0 & -1.26136509985 & 0.00022905610 & -1.26154021709 & 0.00005393885 \\
\hline 0.1 & -1.12965721023 & 0.00005978084 & -1.12960834946 & 0.00001092007 \\
\hline 0.2 & -1.00899116564 & 0.00008507207 & -1.00891722551 & 0.00001113194 \\
\hline 0.3 & -0.89832444563 & 0.00001221659 & -0.89831102836 & 0.00000120068 \\
\hline 0.4 & -0.79665157827 & 0.00005739696 & -0.79670390235 & 0.00000507287 \\
\hline 0.5 & -0.70300414012 & 0.00007531251 & -0.70307995313 & 0.00000050050 \\
\hline 0.6 & -0.61645075626 & 0.00003582923 & -0.61649324757 & 0.00000666206 \\
\hline 0.7 & -0.53609710030 & 0.00003337697 & -0.53606781381 & 0.00000409047 \\
\hline 0.8 & -0.46108589434 & 0.00007992725 & -0.46099764123 & 0.00000832585 \\
\hline 0.9 & -0.39059690898 & 0.00003479547 & -0.39054668049 & 0.00001543301 \\
\hline 1.0 & -0.32384696335 & 0.00018017347 & -0.32404884349 & 0.00002170666 \\
\hline \hline
\end{tabular}




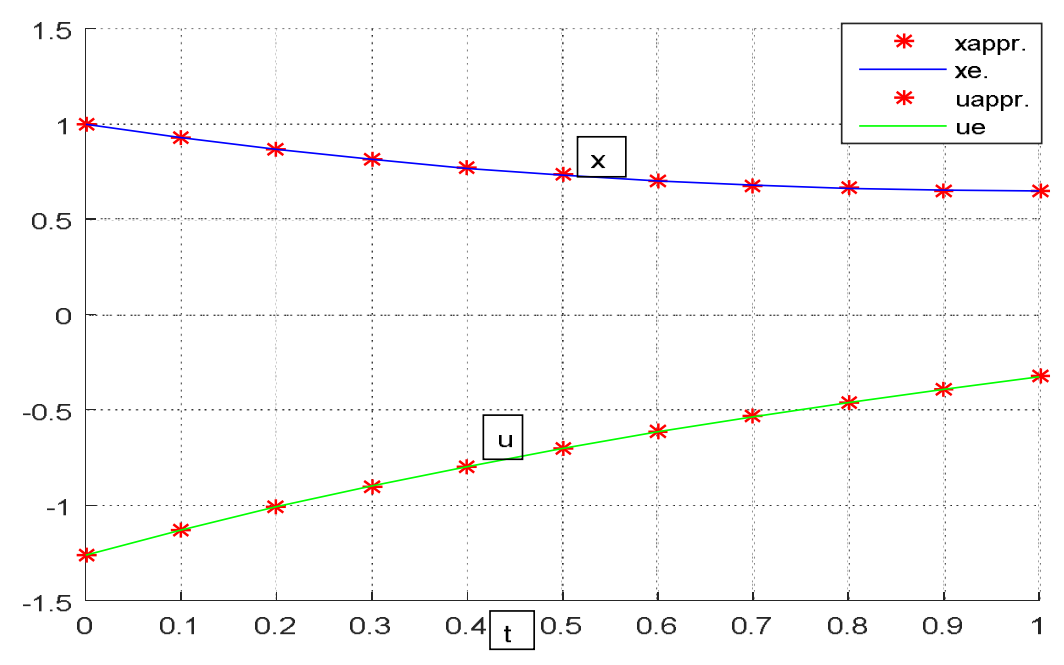

Fig.2 comparison for $\mathrm{x}$ and $\mathrm{u}$ with exact solution for example2

\section{Conclusion}

Utilizing Scaling Boubaker functions as an aid in parameterization techniques in two forms (state, control) were proved to be a powerful tool for solving optimal control problems. Only few number of Scaling Boubaker polynomials were needed to reach a good accuracy. All the approximate results were compared with the exact solution showing good efficiency and capability of this method for solving these problems.

\section{References}

[1] Ahmed I. et al., Indirect Method for Optimal Control Problem Using Boubaker Polynomial, Baghdad Science Journal, Vol.13 (1) (2016).

[2] Colella D. and Heil Ch., Characterizations of Scaling Functions: continuous solutions, SIAM J. MATRIX ANAL. Appl. vol.15 (2), pp. 496 -518 (1994).

[3] Elaydi H., Jaddu H. and Wadi M., An Iterative Technique for Solving Nonlinear Optimal Control Problems Using Legendre Scaling Function, International Journal of Emerging Technology and Advanced Engineering, Vol.2 (6), pp.7-11(June 2012).

[4] Goy T., Combinatorial Determinant Formulas for Boubaker Polynomials, Hindawi, Mathematical Problems in Engineering, Vol.2020, Article, https://doi.org/10.1155 / 2020/1528639.

[5] Gradimir V. and Joksimovic D., Some Properties of Boubaker Polynomials and Applications „Numerical Analysis and Applied Mathematics, AIP Conf. Proc.1479, pp.1050-1053(2012). 
[6] Jaddu H. and Majdalawi A., Legendre Polynomials Iterative Technique for Solving a Class of Nonlinear Optimal Control Problems, International Journal of Control and Automation,Vol.7(3),pp.17-28(2014).

[7] Kafash B., Delavarkhalafi A. and Karbassi S.M., Application of Chebyshev Polynomials to Derive Efficient Algorithms for the Solution of Optimal Control Problems, Scientia Iranica, Computer Science and Electrical Engineering ,Vol.19(3),pp.795-805(2012).

[8] Kafash B. and Delavarkhalafi A., Restarted State Parameterization Method for Optimal Control Problems, Journal of Mathematics and Computer Science,Vol.14, pp.151-161(2015).

[9] Kafash B., Delavarkhalafi A.,Karbassi S. M. and Boubaker K., A Numerical Approach for Solving Optimal Control Problems Using the Boubaker Polynomials Expansion Scheme, Journal of Interpolation and Approximation in Scientific Computing , ID jiasc-00033,Vol.2014 (2014).

[10] Lotfi A., Yousefi S.A. and Dehghan M., Numerical Solution of a Class of Fractional Optimal Control Problems Via the Legendre Orthonormal Basis Combined with the Operational Matrix and the Gauss Quadrature Rule, Elsevier, Journal of Computational and Applied Mathematics,Vol.250,pp.143-160(2013).

[11] Malmir I. , Legendre wavelets with scaling in Time-Delay Systems, Journal Statistic Optimal Inf. Comp.,vol.7, pp.235-253 (March 2019).

[12] Naseif J. and Imad N. Ahmed, Contorllability of Nonlinear Boundary Value Control Systems in Banach Spaces Using Schauder Fixed Point Theorem, Eng. \& Tech. Journal , Vol. 33(4),Part(B), pp.640-653(2015).

[13] Ramezani M., Numerical Solution of Optimal Control Problems by Using a New Second Kind Chebyshev Wavelet, Computational Methods for Differential Equations, Vol.4(2),pp.162169(2016).

[14]Yousfi S. A., Legendre Scaling Function For Solving Generalized Emden-Fowler Equations, International Journal of Information and Systems Sciences, vol. 3(2), pp. 243-250 (2007) . 